\title{
MPPT Of Solar Energy Converter With High-Sensitive Fuzzy PID Controller
}

\author{
ABDULLAH AL GIZI ${ }^{1}$ \\ ${ }^{1}$ Thi-Qar Technical Collage, Electromechanical Engineering Department \\ Thi-Qar technical college, high way street \\ Southern Technical University, IRAQ
}

\begin{abstract}
Progress of the maximum-power-point-tracking (MPPT) structure for solar mounts and rectifiers leftovers stimulating. We recommend a original parametric project of high-sensitive-fuzzy (HSF) ProportionalIntegral-Derivative supervisor (PIDC) for well-organized operative of the MPPT scheme. This project is founded on a synergistic mixture of the genetic-algorithm (GA), radial-basis-function neural-network (RBF$\mathrm{NN}$ ) and Sugeno-fuzzy-logic (SFL) schemes. The finest limits of MPPT and PIDC are strong-minded via optimization, where RBF-NN is adjusted using GA to attain the ideal-solution. Furthermore, RBF-NN is used to augment the PID limits (got from GA) for scheming HSFL-PIDC of the MPPT scheme. The entire scheme is additional tuned by solar strictures underneath numerous working circumstances to recover the solar recital in terms of accusing and correcting. The recital of the proposed analog-implemented MPPT manager is evaluated by applied the system with the dual photovoltaic (PV) system Matlab model. The accomplishment scheme is verified to be efficient and vigorous in refining solar charging and rectifying capacity.
\end{abstract}

Key-Words: - MPPT ,HSF, PIDC, GA,RBF-NN, SFL, HSFL-PIDC

Received: November 18, 2020. Revised: March 6, 2021. Accepted: April 1, 2021. Published: April 18, 2021.

\section{Introduction}

In recent years, dedicated efforts have been made to achieve efficient solar photovoltaic (PV) systems with improved performance. In particular, as a strategy for performance improvement, solar tracking system is researched in-depth. [1], [2], [3], [4] projected a switch procedure that could recover the presentation and dependability of the two-axis solar follower. It was accomplished into two stages including the hardware and software development. Mechanization regulator schemes are widespread in science and skill. The typical hardware device used in manufacturing control is ProgrammableLogic-Controllers (PLC) that panels numerous manufacturing schemes. PLC is browbeaten to regulator plants or manufacturing equipment's such as water and waste switch, energy, oil and gas refining, etc. to cite a few [5]. Usually, a fuzzy PID supervisor is industrialized using PLC for tackling the problematic of a set point weight control in the chief pressure group system. An brainy hierarchical synchronized control plan is positively applied by Hongbo et al. to a $300 \mathrm{MW}$ boiler-turbine unit in China [6]. The philosophy presented by Zadeh deals with the doubt and fuzziness connected info about several limits
[7].The chief impartial of the AVR scheme is to regulator the fatal voltage by regulating the producer exciter voltage. It necessity keep path of the producer fatal voltage all the time below any load disorder by upholding the voltage within predetermined limits [8]. Despite much efforts in emerging progressive control arrangements, the regulator of traditional combined PLC-Fuzzy PID Simulink applied AVR scheme is far from being unspoken [9]. The PID possessing difference, relative and integral constants optimally panels the AVR scheme. Computational practices such as GA and fuzzy logic are used for logical explanation[10]. A tuning fuzzy logic tactic for decisive the best PID manager strictures in AVR scheme is industrialized to obtain on-line PID limits under numerous functioning conditions [11]. The routes of this scheme were strong-minded via optimization. Still, call of Simulink subsystems from MATLAB to STEP 75.5 can be united to PLC [12]. The development of an lively solar panel dual-axis sun next system with all-out power point ensuing fuzzy director was future [13]. This scheme tracked the all-out solar power point and concerned with the solar panel toward the Sun to augment the competence of the PV generation arrangement. The design of a two-axis separate rotary sun tracker [14] was presented and applied. 
Xiaofang and Wencheng [15]applied a new following scheme based on focused photovoltaic (CPV). A taster of two-axis solar subsequent system originated on a PIC microcontroller was also available [16]. The parabolic reflector or parabolic dish is built around two feed diameter to capture the solar liveliness. Dasgupta et al. [17]labeled the design and request of a unique two axis sun subsequent system which used no outdoor light sensors to brand PV cell conflicting in the way of all-out radioactivity to endorse system capability. The applied utilization of solar panels as the devices was the main innovation of the future design. The cross of the solar cells, electrical qualities was used to regulate the insolation limits. Jiao et al. . [18]also future a twoaxis sun-tracking scheme that kept the PV panel vertical to the sun light by complete and relative location sensors signal analysis. We report the project of a HSFL-PIDC and the finest recital in MPPT supervisor system useful for solar vigor conversion and alteration. This afresh planned system mutual the GA, RBF-NN and SFL schemes. The recital of the future employed MPPT supervisor is assessed by The software for the future $\mathrm{RBF}$ and $\mathrm{GA}$ is printed using MATLAB simlink and done on a laptop Intel core(TM)2 Duo CPU 5550@1.83GHz.

\section{Problem Formulation \\ 2.1 RADIAL BASIS FUNCTION NETWORKS}

Moody et al. projected a feed-forward twolayered RBF neural-network with a singlehidden-layer to impersonator the methodical preparation of preventive change in the human mind [19]. The RBF -NN foodstuffs the sturdiest reply near the center of the Gaussian-kernelfunction where each hidden node in the input data space can be stared as a local sensor and the RBF -NN is considered as local approximation model for the skillful processes [20-22].. The diagram of radial-basis purpose neural network is shown in Figuer 1. The updating procedure for the adaptive PID based RBF can be expressed as,

$$
\begin{array}{r}
\Delta k_{p}=\mu \cdot e(k) \cdot e_{p}(k) \cdot \sum_{j=1}^{m} w_{j} h_{j} \frac{c_{j 3}-u(k)}{\sigma_{j}^{2}} \\
\Delta k_{i}=\mu \cdot e(k) \cdot e_{i}(k) \cdot \sum_{j=1}^{m} w_{j} h_{j} \frac{c_{j 3}-u(k)}{\sigma_{j}^{2}}
\end{array}
$$

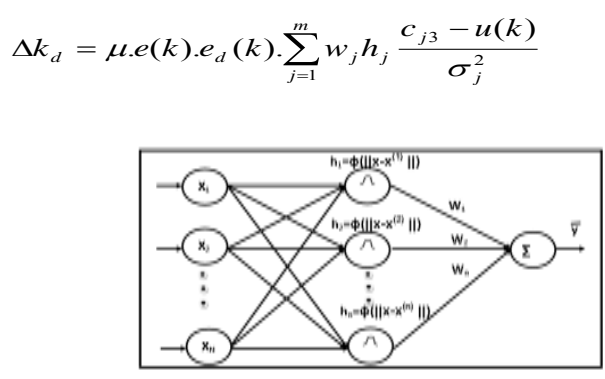

Fig.1.Schematics of RBF neural network structure.

The PID limits such as essential gain (Ki), the relative gain $(\mathrm{Kp})$ and the copied gain $(\mathrm{Kd})$ are mechanically readjusted by RBF on-line knowledge algorithm to uphold the scheme error $=0$. Two instructions offered by Matlab namely Newrb and newrbe are used to design the RBF neural network in which Newrb adds neurons step by step until the goal is hit with long exercise time with minimal error and newrbe designs a network very rapidly with zero error $[23,24]$. In the exercise process, the attained steps are: (i) neurons number in the hidden layer, (ii) the coordinates of the center of RBF function (iii) and the radius (spread) of each RBF functions in each dimension.

\subsection{MPPT}

\subsubsection{Modeling Of Mppt System}

Figuer 2 schematically exemplifies the two-level adaptive switch procedure for the future MPPT [25] scheme. At the first layer, the ripple meaning dominance (RCC) is used to calculate the duty $\mathrm{Hz}$ of the convertor, which is foreseeable to bring all-out available control to the load in the darling state. In the second change level, the faultless orientation adaptive switch (MRAC) building is used to control the delicacies of the converter in reply to the duty bicps intended from RCC, which barred the regalia voltage from fleeting variation after changes in solar insolation.

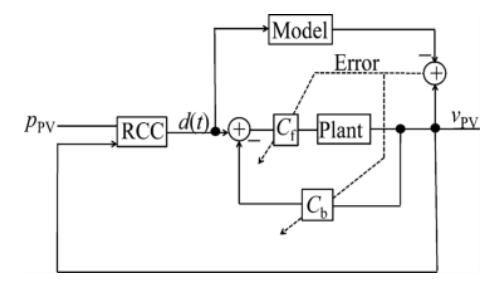

Fig. 2. Schematic diagram of the proposed MPPT system 
As above-mentioned, our newly industrial twolevel MPPT switch process is limited of (RCC) [25-28] in the first level and MRAC [25] in the additional level. Fig. 2 clearly shows that in the first switch level the group voltage VPV and power PPV help as the inputs to the RCC unit. The main innovation of RCC is to use the exchange ripple typical to the converter to worry the scheme and thus path the MPP [25]. The RCC is basically an healthier version of the $\mathrm{P} \& \mathrm{O}$ method [25] except that the alarm is distinguishing to the converter. Besides, RCC has been well-known to meet asymptotically to the MPP with insignificant manager trouble and straight-forward circuit request [25]. The RCC is based on the next comments: the product of the time-based side-shoots of the gathering voltage (V PV) and power (P PV) necessity be (i) greater than zero to the left of the MPP, (ii) less than zero to the right of the MPP, and (iii)precisely zero at the MPP obeying the regulator laws [25]:

$$
\begin{aligned}
& \frac{d p_{p v}}{d t} \frac{d v_{p v}}{d t}>0 \text { when } V_{P V}<V_{M} \\
& \frac{d p_{p v}}{d t} \frac{d v_{p v}}{d t}<0 \text { when } V_{P V}>V_{M} \\
& \frac{d p_{p v}}{d t} \frac{d v_{p v}}{d t}=0 \text { when } V_{P V}=V_{M}
\end{aligned}
$$

These observations lead to the control law derived in[26]

$$
\frac{d d(t)}{d t}=k \frac{d p_{P V}}{d t} \frac{d p_{P V}}{d t}
$$

where $\mathrm{k}$ is a constant called negative gain.

Referring to Fig.2, the regulator law in reckoning (7) can be qualitatively labeled as shadows: if vPV upsurges and there is a following upsurge of $\mathrm{PV}$, the arrangement's employed point is to the left of the MPP and so d should discount, causing an increase of $\mathrm{vPV}$. If $\mathrm{pPV}$ reductions after an upsurge in $\mathrm{vPV}$, then the scheme's working point is to the right of the MPP and thus d requirement increase in order to decrease $\mathrm{v}$ PV. Exploratory equations (6) and (7), one can set the time-based copied of $\mathrm{d}$ to zero so that all-out power is attained. Fig. 3 displays PV the boost converter scheme.

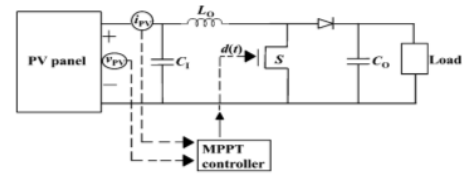

Fig. 3. Circuit diagram of MPPT controller designed for the PV boost converter system

\subsubsection{Proposed MRAC Method}

Fig. 4 shows the future building of MRAC scheme. The contribution to the general scheme, $r$ (t), is the alteration in duty cycle as intended earlier by means of RCC. The effort model agrees to the carry-over purpose (equation 5). Though, for suitability the sign is altered by increasing (-single) so that the vegetable model has only optimistic constant. The input and output of the plant are meant by up (t) and yp (t), correspondingly. Table 1 précises the time value of used boot converter limit. Table 2 enlists the values of adaptive supervisor limits Table 3 compares the values of insignificant phrase with real supervisor limits. Table 4 précises the various transmission purposes and the variety of limit values for solar mechanisms

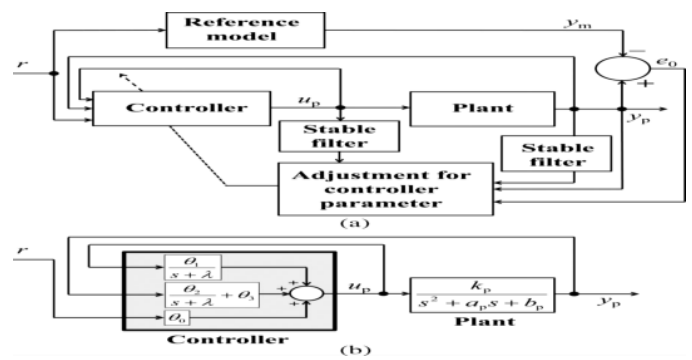

Fig. 4. Diagram performance of the planned: (a) MRAC structure and its (b) controller architecture

Table 1 Values of boost converter parameter

\begin{tabular}{|c|c|}
\hline Circuit parameter & Value \\
\hline RI & $45 \Omega$ \\
\hline LO & $600 \mu \mathrm{H}$ \\
\hline CI & $100 \mu \mathrm{F}$ \\
\hline VO & $350 \mathrm{~V}$ \\
\hline
\end{tabular}


Table 2 Values of adaptive supervisor limits

\begin{tabular}{|c|c|}
\hline Parameter & Value \\
\hline $\mathrm{kp}=\mathrm{Vo} /(\mathrm{Lo} \times \mathrm{CI})$ & $5.83 \times 109 \mathrm{~V}(\mathrm{rad} / \mathrm{sec}) 2$ \\
\hline $\mathrm{ap}=1(\mathrm{R} 1 \times \mathrm{CI})$ & $222 \mathrm{rad} / \mathrm{sec}$ \\
\hline $\mathrm{bp}=1 /(\mathrm{Lo} \times \mathrm{CI})$ & $1.67 \times 107(\mathrm{rad} / \mathrm{sec}) 2$ \\
\hline $\mathrm{km}$ & $5.83 \times 109 \mathrm{~V}(\mathrm{rad} / \mathrm{sec}) 2$ \\
\hline $\mathrm{am}$ & $8.17 \times 103 \mathrm{rad} / \mathrm{sec}$ \\
\hline $\mathrm{bm}$ & $1.67 \times 107(\mathrm{rad} / \mathrm{sec}) 2$ \\
\hline$\kappa$ & 1 \\
\hline $\mathrm{g}$ & 1 \\
\hline$\Gamma$ & $5 \times$ identity matrix \\
\hline
\end{tabular}

Table 3 Contrast between the values of nominal and actual

\begin{tabular}{|c|c|c|c|c|}
\hline & $\Theta 0$ & $\Theta 1$ & $\Theta 2$ & $\Theta 3$ \\
\hline & 1.00 & $-7.95 \times 103$ & -22.8 & $-3.00 \times 10-4$ \\
$\begin{array}{c}\text { Nominal } \\
\text { controller } \\
\text { parameters }\end{array}$ & 1.00 & $-8.12 \times 103$ & -20.4 & $-2.80 \times 10-4$ \\
\hline $\begin{array}{c}\text { Updated } \\
\text { controller } \\
\text { parameters }\end{array}$ & & & & \\
\hline
\end{tabular}

Table 4 Various transmission functions and the range of limit values for solar components

\subsubsection{Optimization of Manager Limits}

The satisfactory process of the scheme is strongminded by the option of the finest PID control limits. Moreover, the selection difficult of the PID manager limits is careful as an optimisation problematic. The neutral function output,

$$
\operatorname{MinF}\left(\mathrm{K}_{\mathrm{d}}, K_{p}, K_{i}\right)=\left(1-e^{-\beta}\right)\left(O_{s h}+E_{s s}\right)+e^{-\beta}\left(t_{s}-t_{r}\right)
$$

The $\operatorname{MinF}(\mathrm{Kp}, \mathrm{Kd}, \mathrm{Ki})$ syndicates transient reply counting rise metre pass, settling time and steady -body politic error. The gratification of the fashionable needs can be attained by choosing appropriate value of the allowance factor $\beta$. Therefore, the optimisation problematic boils down to the next restrictions,

$$
K_{p}^{\min } \leq K_{p} \leq K_{p}^{\max }, K_{i}^{\min } \leq K_{i} \leq K_{i}^{\max }, K_{d}^{\min } \leq K_{d} \leq K_{d}^{\max }
$$

Subsequent Devaraj et al. [29], GA is applied to improve the values manager limits and the future

\begin{tabular}{|c|c|c|}
\hline Components & Transfer function & Parameter limits \\
\hline Plant & 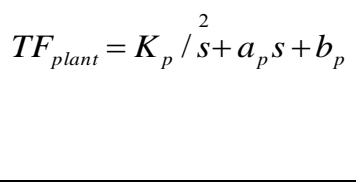 & $\begin{array}{c}K_{p}=V_{O} /\left(L_{O} \times C_{1}\right)_{5.83 \times 109 \mathrm{~V}(\mathrm{rad} / \mathrm{sec}) 2} a_{p}=1 /\left(R_{1} \times C_{1}\right)_{222} \\
(\mathrm{rad} / \mathrm{sec}) 2 b_{p}=1 /\left(L_{O} \times C_{1}\right)_{1.67 \times 107(\mathrm{rad} / \mathrm{sec}) 2 k_{m}, b_{m}, \lambda g, \Gamma} g, \Gamma(\mathrm{rad} / \mathrm{sec}) 2,8.17 \times 103 \mathrm{rad} / \mathrm{sec}, 1.67 \times 107(\mathrm{rad} / \mathrm{sec}) 2,1,1,5 \\
\text { identity matrix }\end{array}$ \\
\hline Controller in $r$ & $\theta_{r}=0.5\left(\lambda+\theta_{0}\right)$ & $0<\theta_{O} \leq 1 \lambda=1$ \\
\hline Controller feedback $y_{p}$ & $y_{p}=\left(\theta_{2} / s+\lambda\right)+\theta_{3}$ & $\begin{array}{c}-22.8 \leq \theta_{2} \leq-20.4 \\
-3 \times 10^{-4} \leq \theta_{3} \leq-2.8 \times 10^{-4}\end{array}$ \\
\hline Controller $\boldsymbol{u}_{P}$ & $u_{p}=\theta_{1} / s+\lambda$ & $-7.95 \times 10^{3} \leq \theta_{1} \leq-8.12 \times 10^{3} \theta_{1} \cong-8.035 \times 10^{-3}$ \\
\hline
\end{tabular}
GA is obtainable. 


\subsubsection{Proposed GA}

GA is documented as an real and efficient method to solve the optimization glitches. In contrast to the optimization methods, such as chance search and replicated hardening, GA presentation is superior that avoids local minima careful as a key issue in nonlinear schemes $[10,11]$.

\subsubsection{Genetic Algorithm Operators}

The major GA workers such as cross-over, imitation and change are exploited. The meeting speed is skillful by applying various likelihoods on these workers. The project of the cusp and change operators are prudently achieved due to their huge impact on the presentation of geneticalgorithm [10, 11]. The details of the genetic workers used in the projected GA are illustrated in Table 4.

\subsubsection{Reproduction}

In the procedure of imitation, persons are selected contingent on their fitness purpose, the higher the fitness is, more chance for an separate to be designated for the next cohort. Three main selection approaches such as position method, fitness stable assortment and contest selection are utilized [29]. In this effort, we employ the contest selection technique, where ' $n$ ' persons are randomly designated from the populace and the finest vale is chosen for extra genetic dispensation. This procedure is recurrently done until the breeding pool is filled.

\subsection{Crossover}

The stuff of worldwide search in GA is typically strong-minded by the cusp worker, which organizations two-parent genes to crop a new one. The diversity of the chosen likelihood is typically amid $0.6-1.0$. One of the stimulating topographies of the cusp workers is the relation amid the made DNA and the location of both the parentages. The made new chromosome leftovers close to the parents in case both the parentages are close to each other. Equally, the search is more probable to be chance [29].

\subsection{Mutation}

New chromosome is presented into the public for the alteration process. Change randomly makes an insignificant variety in the gene info. However, for random alteration, the changeable takes a dependable chance number among the lower and higher limits. In this study 'static mutates' employee is used.

\subsubsection{GA Application is adjusting limits of MMPPT PID}

The best PID controller limits are got via GA tuning of PID. Two main melodies such as sign of the excellent variables (variable symbol) and preparation of the fitness function are used in this procedure.

\subsubsection{Variable Representation}

The answers of all applicants are made in the genetic populace. The answer elements of PID controller-tuning problematic comprise limits $\mathrm{Ki}$, $\mathrm{Kp}$ and $\mathrm{Kd}$. The direct picture of the answer variables decreases the processor space for storage the populace. The values of these limit got from direct change of GA into the RBF package for the best tuning of the PID manager limit are considerable for the thematic factory process of MMPPT scheme.

\subsubsection{Fitness Function}

The answer for the performance of every candidate in the public is assessed founded on its appropriateness which is clear as a non-negative worth to be browbeaten. Fitness is related in a conformist line with the value of neutral function. The limit set of the separate valuation can be a strong-minded using calculation (6) for the presentation criteria. The value of distinct fitness is intended by the importance of the presentation criteria via mutual calculation. The appropriateness, purpose is the presentation of the sympathy criterion $\mathrm{F}(\mathrm{KP}, \mathrm{Kd}, \mathrm{Ki})$ given in equation (6). Thus, the minimization of 
performance criteria in eq. (6) can be distorted to the growth of the fitness function as,

$$
\text { Fitness }=\frac{k}{F\left(K_{\mathrm{d}}, K_{p}, K_{i}\right) * \text { ITAE }}
$$

Where $\mathrm{k}$ is a continuous, ITAE is a time vital augmented by the total error value. This is used to strengthen the value of $1 / F$, which is typically small, so that, the hereditary material appropriateness values happen in a wider diversity.

\subsection{Sugeno Fuzzy Model}

Newly, the fuzzy set philosophy was used in which a adaptable is a subordinate of one or more sets with a association stated degree [29] . The fuzzy rule can be spoken as:

$$
\text { If } x \text { is } A \text { and } y \text { is } B \text { then } z=f(x, y)
$$

anywhere A and B are fuzzy groups in the forerunner, $\mathrm{x}$ and $\mathrm{y}$ are the participation variables and $\mathrm{f}(\mathrm{x}, \mathrm{y})$ is a crisp occupation in the subsequent.

Originally, the least of the connotation jobs input $\left(w_{i}\right)$ is got for each rule, wherever this value is the gunfire value for a exact rule. Also, the general output is envisioned by a biased average of distinct rule manufactures using the preparation:

$$
z=\frac{\sum_{i=1}^{M} \omega_{i} z_{i}}{\sum_{i=1}^{M} \omega_{i}}
$$

The bounds of PIDC under numerous working settings are strong-minded by the SF scheme.

\subsection{MYTHOLOGY TO DESIGN FUZZY PID CONTROLLER \\ 3.1 Development of a Sugeno Fuzzy Model to Design PID controller}

The applied and last border to loop performance can be obedient by sympathetic an indirect deceased time $(\theta \mathrm{r})$ from the change relative $r=\theta r=0.5\left(\lambda+\theta_{0}\right)$. The deceased time can be approached as the unique deceased time $(\theta 0)$ increased by a issue of 0.5 to the shut loop time constant $(\lambda)$ for a setpoint alteration. For extreme trouble refusal by a PID, value of $\lambda$ is set equal to the unique deceased time. In this state, the deceased time is equal to the unique deceased time. $r=\theta r=0.5\left(\lambda+\theta_{0}\right), \theta \mathrm{o}=1, \lambda=1$. The top and combined errors for unmeasured step turbulences represents the worst case. The ideal PID limits for real procedure are got by evolving SF logic perfect, where, $\theta \mathrm{r}$ and yp $(\theta 1, \theta 2)$ are the aids with $\mathrm{Kp}, \mathrm{Kd}$ and $\mathrm{Ki}$ are the outputs. Eight fuzzy sets such as 'very low (VL)', 'low(L)' ,'medium low (ML)', 'medium(M)', 'medium high (MH)' ,'high low (HL)', 'high medium (HM)' and 'high (H)' are clear for the mutable Or . Likewise, the fuzzy sets clear for the mutable yp $(\theta 1, \theta 2)$ are 'very low (VL)', 'low (L)', 'medium low (ML)', 'medium high (MH)', 'high (H)' and 'very high (VH)'. They are connected with meeting three-sided participation purposes. To express the table for fuzzy rule, the values of Or are diverse from 0.7 to 1.0 in steps of 0.1 and the values of yp are numerous from 1 to 2 in steps of 0.2. For each mixture of $\Theta r$ and yp, the future RBF rule via GA is practical to get the best values of $\mathrm{Kp}, \mathrm{Kd}$ and $\mathrm{Ki}$ in each times. The fuzzy rule is spoken for $\mathrm{Kp}, \mathrm{Kd}$ and $\mathrm{Ki}$ and shortened in Table 5(a)-(c), congruently. Throughout real-time procedure, the values of $\Theta r$ and yp are strongminded. Using these values of Or and yp, the best value of $\mathrm{Kp}, \mathrm{Kd}$ and $\mathrm{Ki}$ are intended by the fuzzy rule table and the FIS editor Surgeon implication system. Depending on the initialization (FIS editor), the values of inputs of the fuzzy logic boss are $\Theta r$, yp and the outputs are $(\mathrm{Kp}, \mathrm{Kd}$ and $\mathrm{Ki})$. 
Table 5 Limits used to generate the Sugeno fuzzy rule

\begin{tabular}{|c|c|c|c|c|c|c|}
\hline & Very Low & Low & Medium Low & Medium High & High & Very high \\
\hline$r_{=} \theta_{r}=0.5\left(\lambda+\theta_{0}\right)$ & 0.1 & 0.2 & 0.4 & 0.6 & 0.8 & 1 \\
\hline $\begin{array}{c}y_{p},-22.8 \leq \theta_{2} \leq-20.4 \\
-3 \times 10^{4} \leq \theta_{3} \leq-2.8 \times 10^{4}\end{array}$ & 0.55 & 0.6 & 0.7 & 0.8 & 0.9 & 1 \\
\hline \multicolumn{6}{|c|}{$u_{p}=\theta_{1} / s+\lambda, \theta_{1} \cong-8.035 \times 10^{3}$} & \\
\hline \multicolumn{7}{|c|}{ For proportional gain $K_{p}$} \\
\hline Low $(-22.8)-3,0.55$ & 0.7947 & 0.7253 & 0.9814 & 0.8045 & 0.7292 & 0.9062 \\
\hline Medium low $(-22)(-2.933)$ & 0.9804 & 0.9822 & 0.5904 & 0.7195 & 0.9912 & 0.9541 \\
\hline Medium high $(-21.2)(-2.866)$ & 0.9062 & 0.6500 & 0.6520 & 0.8759 & 0.8416 & 0.6931 \\
\hline $\operatorname{High}(-20.4)(-2.8)$ & 0.8651 & 0.9599 & 0.7977 & 0.9951 & 0.6197 & 0.8192 \\
\hline \multicolumn{7}{|c|}{ For integral gain $\boldsymbol{K}_{\boldsymbol{i}}$} \\
\hline Low $(-22.8)$ & 0.4946 & 0.4990 & 0.4585 & 0.4961 & 0.4990 & 0.4804 \\
\hline Medium low(-22) & 0.5000 & 0.4883 & 0.4717 & 0.4907 & 0.4812000 & 0.4971 \\
\hline Medium high $(-21,2)$ & 0.4966 & 0.4861 & 0.4717 & 0.4995 & 0.4589 & 0.4834 \\
\hline $\operatorname{High}(-20.4)$ & 0.4980 & 0.4844 & 0.4941 & 0.4990 & 0.4726 & 0.4956 \\
\hline \multicolumn{7}{|c|}{ For derivative gain $K_{d}$} \\
\hline Low $(-22.8)$ & 0.0428 & 0.0352 & 0.0757 & 0.0229 & 0.0150 & 0.0101 \\
\hline Medium low(-22) & 0.0010 & 0.0199 & 0.18443 & 0.0000 & 0.00110 & 0.0528 \\
\hline Medium high $(-21,2)$ & 0.0613 & 0.1674 & 0.0126 & 0.0463 & 0.00422 & 0.1076 \\
\hline $\operatorname{High}(-20.4)$ & 0.01660 & 0.0029 & 0.00023 & 0.0067 & 0.1049 & 0.270 \\
\hline
\end{tabular}

The scheme with three fuzzy logic controllers (Kp, $\mathrm{Kd}$ and $\mathrm{Ki}$ ) and rule viewer are documented, in which each supervisor has two inputs (Or, yp) and each input has fuzzy recognized associated with it. The input variety for $\Theta r$ is $0.7 \leq \Theta r \leq 1$ and yp is $0.4 s<y p<1 s$. The output standards of $\mathrm{Kp}, \mathrm{Kd}$ and $\mathrm{Ki}$ be depending on the change of Or with respect to yp finished the output setting rule recognized on the tabularized values. The output 
has 72 fuzzy set rules for $\mathrm{Kp}, \mathrm{Kd}$ and $\mathrm{Ki}$, and 48 rules for each one limit as portrayed in Fig. 5 rule viewer. For this value of $\mathrm{Or}$ and yp, the finest value of $\mathrm{Kp}, \mathrm{Kd}$ and $\mathrm{Ki}$ can be calculated using the fuzzy rule table and the sugeno implication scheme. The best results are got with the next regulator limits for GA: the number of generation $=50$, population size $=30$, crossover $=0.6$, mutation probability $=0.001$. Also, by choosing the correct value of the weighting factor $\beta=1$, the presentation criterion can be made to gratified the designer duty. The software for the future $\mathrm{RBF}$ and GA is written using MATLAB and done on a laptop Intel core(TM)2 Duo CPU 5550@1.83GHz. The GA disbursed $23.79 \mathrm{~s}$ to reach the best solution.

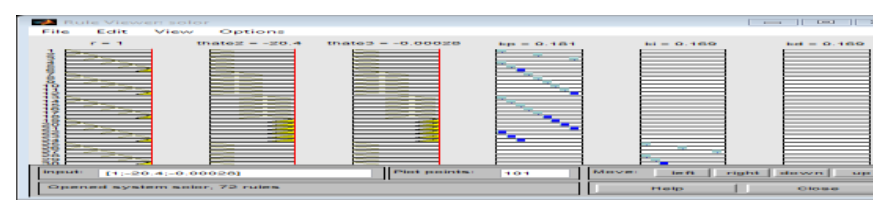

Fig. 5. Computer screen shot (the rule viewer) for the planned Fuzzy organizer of sun tracker system.

\subsection{Control of PV Power Using HSFL-PIDC}

Fig. 6 portrays the circuit illustration to control the PV power using FLC. Fig. 7 exemplifies the circuit illustration of the future Simulink with the fuzzy manager free as sun tracker scheme. The manager has been tested using a Simulink motor component in MATLAB, by applying the step input and initial degree of the rotor of -10 degree. Fig. 8 shows the output step answer in the range of -10 to 0 degrees, which is attained in 5 steps with each step in the motorized of 1.8 degrees. Fig. 9(a)-(c) shows the reply of PV with fuzzy manager output at dissimilar time and the $\mathrm{Vpv}$ output

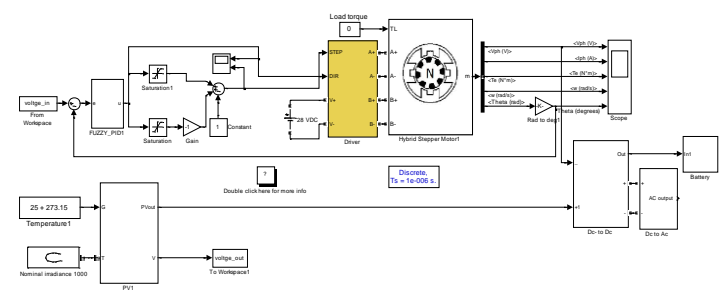

Fig. 6. Circuit diagram revealing the regulator of PV power using FLC.
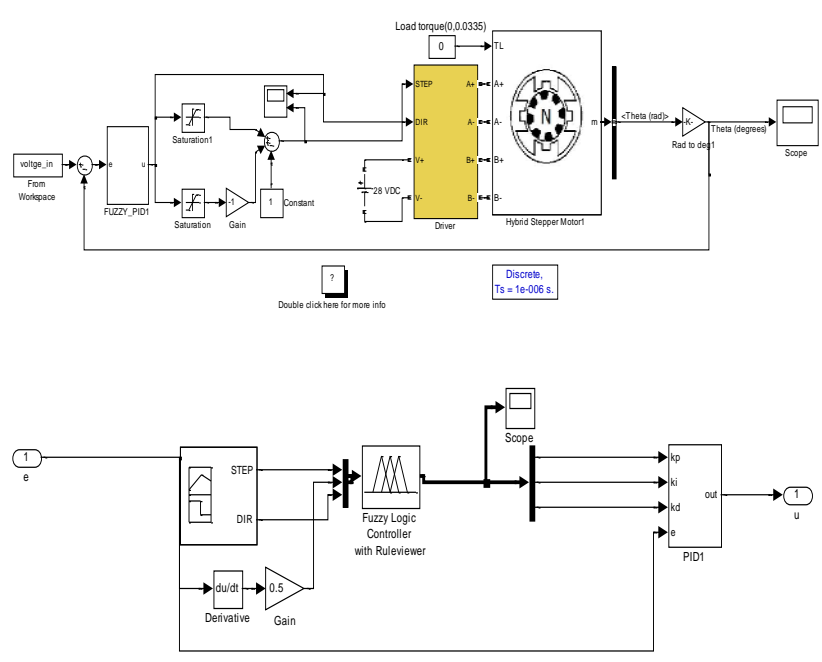

Fig. 7. Simulink block diagram for the fuzzy manager for sun tracker system.

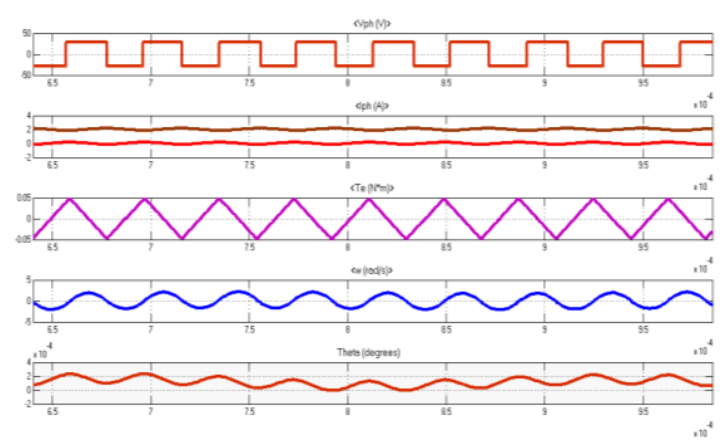

Fig. 8. The output of the driver.
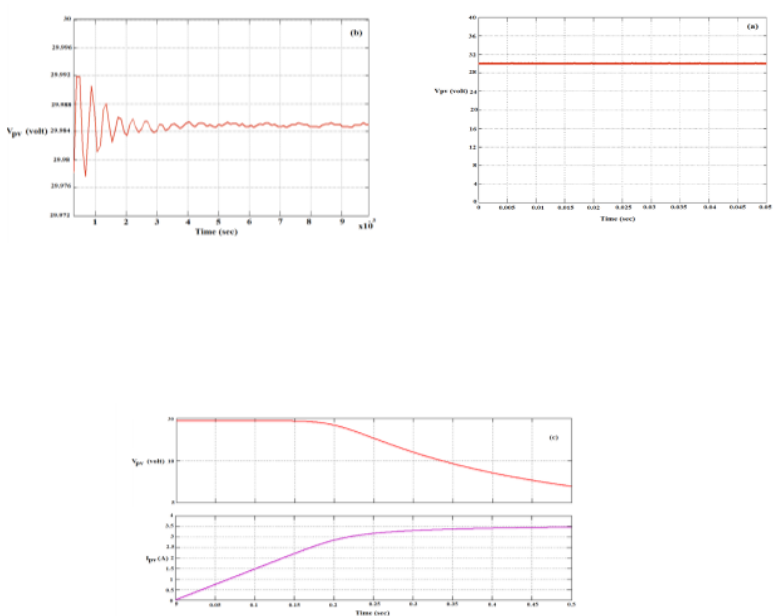

Fig. 9. The response of PV with fuzzy manager output at (a) $0.05 \mathrm{~s}$, (b) $0.001 \mathrm{~s}$, and (c) the $\mathrm{V}_{\mathrm{pv}}$ output. 


\subsection{MPPT of PV Using HSFL-PIDC Implementation}

In this imitation, originally the battery is first cleared and then invigorated both at a continuous current of $5.5 \mathrm{~A}$. A simple current model is used to duplicate the cordless fever. It is assumed that cooling is chiefly via convection, and the heating is majorly since the battery interior resistance (R2). A normal $14 \mathrm{~V}$ lead-acid battery is demonstrated by linking seven copies of the $2 \mathrm{~V}$ cordless cell block in series[30]..

The best PID limits of the fuzzy inference is carried out via real-time process and got by emerging SFL model for the MPP tracker as showed in Fig. 10(a,b,c). It is obvious that the signals are not flat but they carried a constituent of the all-out power amid voltage and current. The energy range altered from $24 \mathrm{~V}$ to $32 \mathrm{~V}$ and the current IL is varied amid 0 and $10 \mathrm{~A}$ during the inductance and $-5,+5$ as during the capacitor at time $0.05 \mathrm{sec}$. The voltage signal (Fig.11) is ranged amid $26-32 \mathrm{~V}$ at gazing and stable state at $28.6 \mathrm{~V}$ similar to the orientation signal, where the current signal loomed is varied between $4.8-13$ at staring and steady sate at $5.5 \mathrm{~A}$ alike to the reference signal showed this. The fuzzy logic supervisor with PWM signal has two compensations to the inverter. First, it crops a smooth error-free sine-wave. Second, it achieves a smooth change for the current sign and constant (no) change for the voltage signal (in variableload case). The smooth transition saves the load from destruction by high-voltage pulses or turbulences. Fig. 12 shows the inverters voltage for mutable unity power factor load.

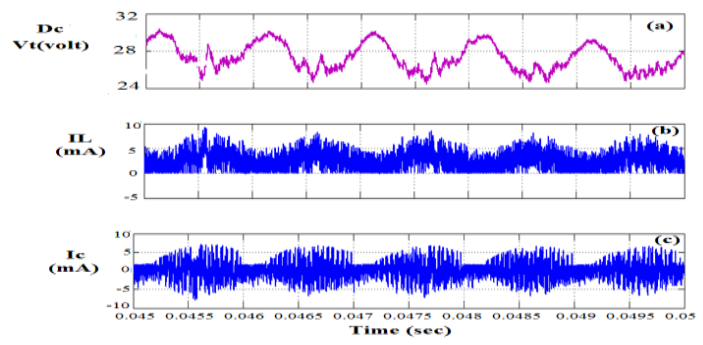

Fig. 10. DC-DC output with FC
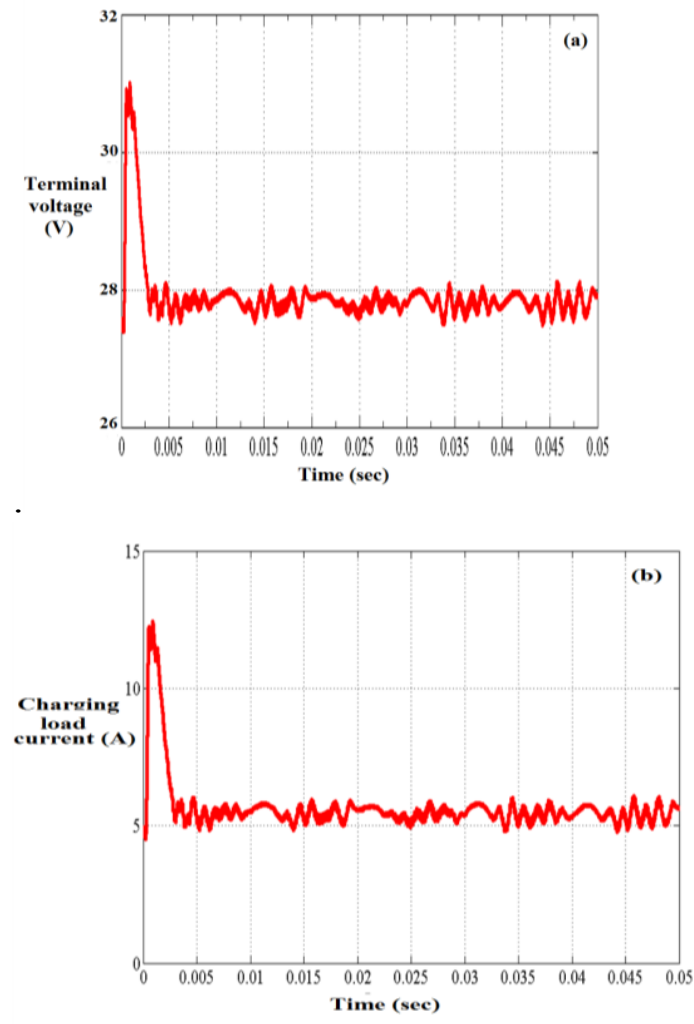

Fig.11. The battery charging output.

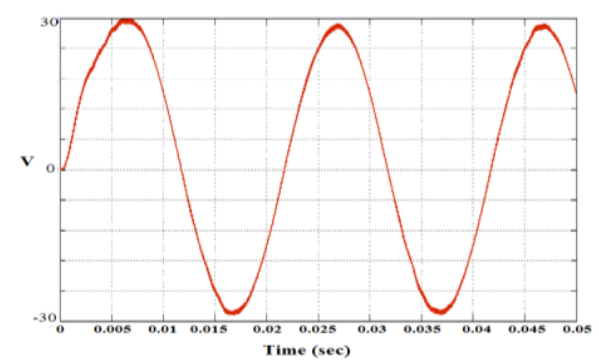

Fig. 12. AC output

Dependability of the planned HSFL-PID controller with MPPT voltage limits is checked and likened with existing manual and Arduino following voltage. It is experiential that the answer of MPPT scheme with new HSFL-PIDC supervisor is highly subtle for a actual minor alteration as depicted in Fig. 13. By likening our results with the following voltage of Arduino manual and HSFL-PIDC it is found that the proposed HSFL-PID controller has least settling time, less rise time and steady-state error as showed in Fig. 9(a). However, the replies observed from the present manager have a slight 
overshoot which can be further better-quality by cumulative the number of rules.

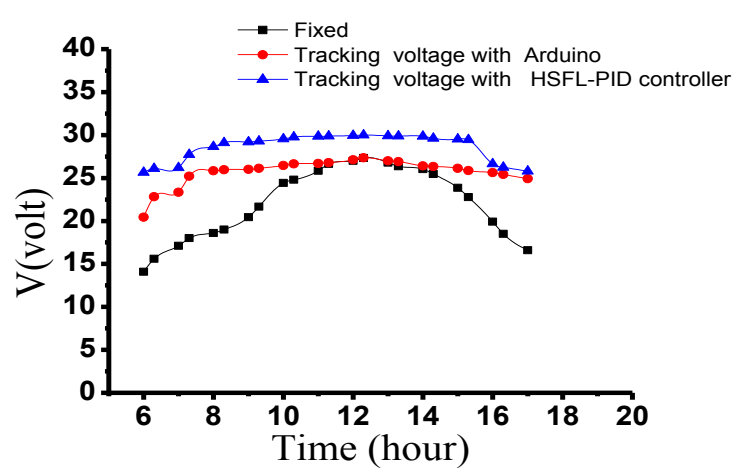

Fig. 13. Seen comparison of overall method of Fixed (manual tracking), Following voltage with Arduino and Tracking voltage with HSFL-PID manager

Fig. 14 likens the overall presentation of MPPT method with ANN [31], PSO [31], and theoretic HSFL-PIDC. It is evident that the future HSFLPIDC located the all-out power point (MPPT) of the PV module very close to the theoretic value as likened to the traditional PSO procedure and ANN.

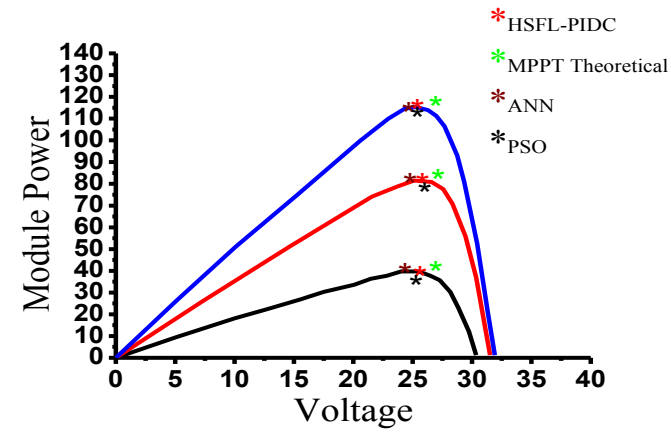

Fig. 14. Likening P-V physiognomies of different approaches.

Fig. 15 shows the battery accusing voltage difference of the 1A-3P PV, the secure PV and following with HSFL-PIDC throughout the day. It is experiential that the battery voltages of both sLED are very close and continuously beneath the overprice point (28.6 V) [32], and with HSFLPIDC beneath the overcharge point (28.61652). This brands the contrast of PV power cohort, i.e. the charged vigor to the battery, to be on a shared basis and further precise. The battery voltage of the fixed PV is a slight bit lesser than that of the 1A-3P PV temporarily the power cohort of the fixed PV is less than the 1A-3P PV, whereas the proposed HSFL-PIDC contrast with overall approaches gives development of MPPT [32].

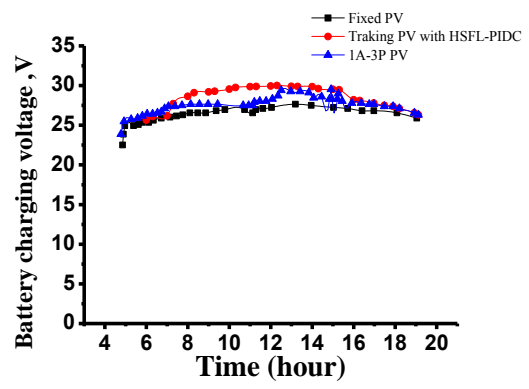

Fig. 15. Comparison of battery charging voltage of overall method of Fixed PV [32], tracking voltage with $1 \mathrm{~A}-3 \mathrm{P} \mathrm{PV}[32]$ and tracking voltage with HSFL-PIDC.

The competence of classical PSO procedure is calculated by in-between the got power by the theoretical all-out power of PV module. Equally, the efficiency of the future procedure is obtained by dividing the foretold power by the theoretical all-out power of PV module. Rendering to the results, the following efficiency of the future algorithm is not less than $96 \%$ as likened to the classical $\mathrm{P} \& \mathrm{O}$ procedure and $\mathrm{ANN}$ as shown in Fig. 16 Thus, the future HSFL-PIDC algorithm is efficient under dissimilar values of solar radiation.

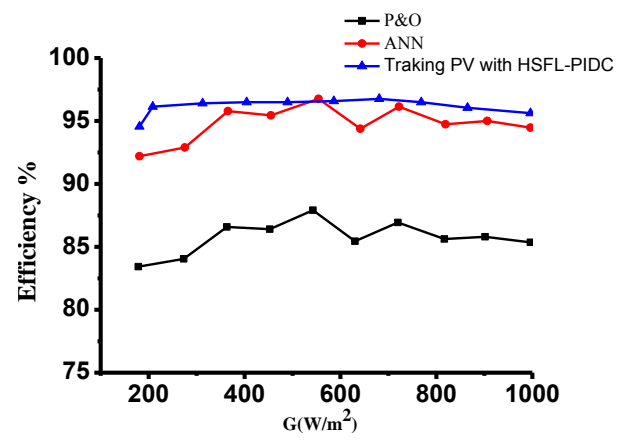

Fig. 16. Comparing efficiency of the proposed PV system with other methods. 
Table 6 Three Months total power generation comparing with proposed methods

\begin{tabular}{|c|c|c|c|}
\hline Month & 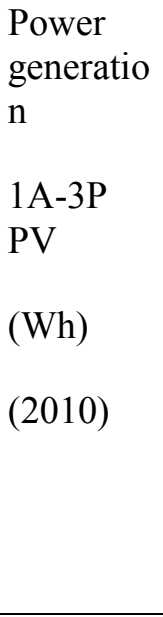 & $\begin{array}{l}\text { Tracking } \\
\text { voltage with } \\
\text { HSFL-PID } \\
\text { controller } \\
\text { PV (Wh) } \\
(2016)\end{array}$ & $\begin{array}{l}\text { Increase of } \\
\text { PV power } \\
\text { Generation } \\
\left(E_{\text {inc }}\right),(\%) \\
E_{\text {Inc }}=(H S F L- \\
\text { PID } \\
\text { controller } \\
\text { PV- power } \\
\text { generation of } \\
1 \text { A-3P PV)/ } \\
\text { power } \\
\text { generation of } \\
\text { 1A-3P PV }\end{array}$ \\
\hline March & 28,044 & 36.04792 & 28.54 \\
\hline April & 17,136 & 36.55208 & 113.30 \\
\hline May & 25,082 & 37.8125 & 50.76 \\
\hline Total & 70.262 & 110.4125 & 64.20 \\
\hline
\end{tabular}

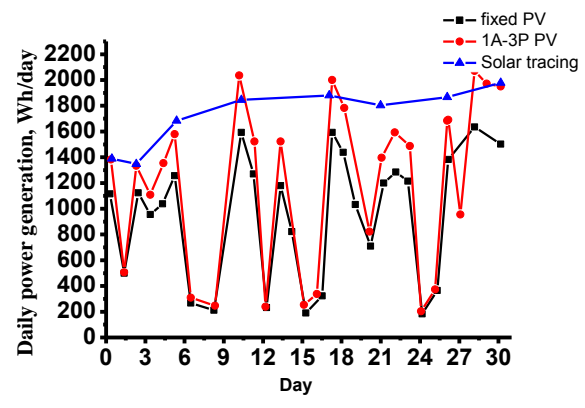

Fig. 17. Long-term test results of 1A-3P tracking PV, fixed PV (March, 2010) and tracking voltage with HSFL-PIDC.

The daily presentation in March shows that the upsurge of solar deceiving with HSFL-PIDC power generation upsurges with daily solar irradiation likened with 1A-3P PV and fixed PV. The difference of daily power cohort is increased of solar tricking with HSFL-PIDC with solar radioactivity in the long-term test. It is seen that power cohort increase greater than $30.1829 \%$ can be predictable in sunny days $[32,33]$.

The once-a-month test results are abridged in Table 6. The upsurge of monthly-total power generation from HSFL-PIDC sun following PV is observed to be in amid $28.54 \%$ and $113.30 \%$. The total power cohort is increased in the test retro from March 1, 2016 to March 31, 2016, is $64.20 \%$ which is greater than to the theoretic forecast of solar energy capture $(24.5 \%)$ by Huang and Sun (2007).Chang (2009) done a theoretical calculation of yearly gains for a single-axis continuous following PV installed at Taichung, central Taiwan, with the yearly optimal tilt angle (latitude) $[32,33]$.

\section{Conclusion}

To recover the vigor gain of solar power florae a new solar following scheme is projected and realized. This dual-axis following organization attained the highest energy gain in every region and obtainable as the most multipurpose scheme. Moreover, results exposed that it can be connected anywhere with certain high vigor gain. HSFL-PIDC manager is used to regulator the position which safeguarded the suitable position on the panel and improve the solar recital in terms of charging and correcting. The act of the projected analog-implemented MPPT with HSFLPID manager is appraised by associating with other connected works. The projected automatic solar following system is established to achieve an overall power upsurge more than the fixedangle $\mathrm{PV}, 1 \mathrm{~A}-3 \mathrm{P} \mathrm{PV}$, Classical $\mathrm{P} \& \mathrm{O}$ algorithm and ANN. The attainment system is confirmed to be efficient and robust in cultivating solar charging and correcting capacity.

\section{ACKNOWLEDGEMENTS}

Abdullah is thankful to Dr.S.K.Ghushal for many valuable suggestions and critical readings of the manuscript.

\section{References:}


[1] M. Engin and D. Engin, "Optimization mechatronic sun tracking system controller's for improving performance," in 2013 IEEE International Conference on Mechatronics and Automation, 2013: IEEE, pp. 1108-1112.

[2] M. Zolkapli, S. Al-Junid, Z. Othman, A. Manut, and M. M. Zulkifli, "High-efficiency dual-axis solar tracking developement using Arduino," in Technology, Informatics, Management, Engineering, and Environment (TIME-E), 2013 International Conference on, 2013: IEEE, pp. 43-47.

[3] S. Elagib and N. Osman, "Design and implementation of dual axis solar tracker based on solar maps," in 2013 INTERNATIONAL CONFERENCE ON COMPUTING, ELECTRICAL AND ELECTRONIC ENGINEERING (ICCEEE), 2013.

[4] M. A. J. Paul, "Design and performance analysis of automated two axis solar tracking system for steam generation," in Energy Efficient Technologies for Sustainability (ICEETS), 2013 International Conference on, 2013: IEEE, pp. 432-437.

[5] W. Chen, M. Xing, and K. Fang, "A PLC-based fuzzy PID controller for pressure control in Coke-oven," in Control Conference (CCC), 2012 31st Chinese, 25-27 July 2012 2012, pp. 4754-4758.

[6] H. Liu, S. Li, and T. Chai, "Intelligent coordinated control of power-plant main steam pressure and power output," Journal of Systems Engineering and Electronics, vol. 15, no. 3, pp. 350-358, // 2004. [Online]. Available: http://www.scopus.com/inward/record.url?eid= 2-s2.0-

10044262121\&partnerID $=40 \& \mathrm{md} 5=062 \mathrm{~b} 7 \mathrm{e} 90$ 5f1011da9253b4f7f5bc6f51.

[7] Z. L. A, " Fuzzy sets,Information and Control," vol. 8:338-353, 1965.

[8] N. Farouk and B. Tian, "Application of selftuning fuzzy PID controller on the AVR system," in Mechatronics and Automation (ICMA), 2012 International Conference on, 5-8 Aug. 2012 2012, pp. 2510-2514, doi: 10.1109/ICMA.2012.6285741.

[9] O. D. A, Handbook of PI and PID controller tuning rules. 2003.

[10] R. A. a. D. K. Baskarn, " Speed Control of Induction Motor Using Fuzzy PI and Optimized Using GA," International Journal of Recent Trends in Engineering, vol. 2, no. 5, 2009.
[11] D. P. P. Neenu Thomas, "Position Control of DC Motor Using Genetic Algorithm-based PID Controller," London, U.K Proceedings of the World Congress on Engineering, vol. 2, no. WCE 2009, 2009.

[12] Siemens, SIMATIC WinAC S2O Wizard February 2012

[13] H.-C. Lu and T.-L. Shih, "Fuzzy system control design with application to solar panel active dual-axis Sun tracker system," in Systems Man and Cybernetics (SMC), 2010 IEEE International Conference on, 2010: IEEE, pp. 1878-1883.

[14] A. Al Nabulsi, A. El Nosh, A. Ahli, M. Sulaiman, and R. Dhaouadi, "Efficiency optimization of a 150W PV system using dual axis tracking and MPPT," in Energy Conference and Exhibition (EnergyCon), 2010 IEEE International, 2010: IEEE, pp. 400-405.

[15] S. Xiaofang and G. Wencheng, "A Sun Spot Center Orientation Method Based on Ellipse Fitting in the Application of CPV Solar Tracker," in Intelligent System Design and Engineering Application (ISDEA), 2010 International Conference on, 2010, vol. 1: IEEE, pp. 175-178.

[16] L. L. Oo and N. K. Hlaing, "Microcontrollerbased two-axis solar tracking system," in Computer Research and Development, 2010 Second International Conference on, 2010: IEEE, pp. 436-440.

[17] S. Dasgupta, F. W. Suwandi, S. Sahoo, and S. Panda, "Dual axis sun tracking system with PV cell as the sensor, utilizing hybrid electrical characteristics of the cell to determine insolation," in 2010 IEEE International Conference on Sustainable Energy Technologies (ICSET), 2010: IEEE, pp. 1-5.

[18] H. Jiao, J. Fu, Y. Li, and J. Lai, "Design of automatic two-axis sun-tracking system," in 2010 International Conference on Mechanic Automation and Control Engineering, 2010.

[19] L. Yao-Lun, T. Chia-Chang, J. Wu-Shun, and L. Shuen-Jeng, "Design an Intelligent NeuralFuzzy Controller for Hybrid Motorcycle," in Fuzzy Information Processing Society, 2007. NAFIPS '07. Annual Meeting of the North American, 24-27 June 2007 2007, pp. 283-288, doi: 10.1109/nafips.2007.383852.

[20] S. M. S. S.N. Qasem, "Memetic Elitist Pareto Differential Evolution algorithm based Radial Basis Function Networks for classification problems," Applied Soft Computing, vol. 11, no. 1, pp. 5565-5581., 2011. 
[21] S. M. S. S.N. Qasem, "Radial basis function network based on time variant multi-objective particle swarm optimization for medical disease diagnosis," Applied Soft Computing, vol. 11, no. 1, pp. 1427-1438, 2011.

[22] M. A. M. P. N. A. Al-geelani, R. Q. Shaddad "Characterization of acoustic signals due to surface discharges on H.V. glass insulators using wavelet radial basis function neural networks " Applied Soft Computing, vol. 7, no. 2, pp. 1327-1338, 2012.

[23] H. Sang Jeen, G. S. May, and P. Dong-Cheol, "Neural network modeling of reactive ion etching using optical emission spectroscopy data," Semiconductor Manufacturing, IEEE Transactions on, vol. 16, no. 4, pp. 598-608, 2003, doi: $10.1109 /$ tsm.2003.818976.

[24] M.-W. K. Shu-Kun Zhao, Yi-Seul Han, SeYoun Jeon, Yun-Keun Lee, and Seung-Soo Han, " Radial Basis Function Network for Endpoint Detection in Plasma Etch Process," Springer-Verlag, vol. 67, pp. 253-263, 2010.

[25] R. Khanna, Q. Zhang, W. E. Stanchina, G. F. Reed, and Z.-H. Mao, "Maximum power point tracking using model reference adaptive control," IEEE Transactions on power Electronics, vol. 29, no. 3, pp. 1490-1499, 2014.

[26] P. T. Krein, "Ripple correlation control, with some applications," in Circuits and Systems, 1999. ISCAS'99. Proceedings of the 1999 IEEE International Symposium on, 1999, vol. 5: IEEE, pp. 283-286.

[27] D. Logue and P. Krein, "Optimization of power electronic systems using ripple correlation control: A dynamic programming approach," in Power Electronics Specialists Conference, 2001. PESC. 2001 IEEE 32nd Annual, 2001, vol. 2: IEEE, pp. 459-464.

[28] J. W. Kimball and P. T. Krein, "Discrete-time ripple correlation control for maximum power point tracking," IEEE Transactions on Power Electronics, vol. 23, no. 5, pp. 2353-2362, 2008.

[29] D. Devaraj and B. Selvabala, "Real-coded genetic algorithm and fuzzy logic approach for real-time tuning of proportional-integral derivative controller in automatic voltage regulator system," Generation, Transmission \& Distribution, IET, vol. 3, no. 7, pp. 641-649, 2009, doi: 10.1049/iet-gtd.2008.0287.

[30] R. A. Jackey, "A simple, effective lead-acid battery modeling process for electrical system component selection," SAE Technical Paper, 0148-7191, 2007.
[31] M. A. Younis, T. Khatib, M. Najeeb, and A. M. Ariffin, "An improved maximum power point tracking controller for PV systems using artificial neural network," Przegląd Elektrotechniczny, vol. 88, no. 3b, pp. 116-121, 2012.

[32] B. Huang, W. Ding, and Y. Huang, "Long-term field test of solar PV power generation using one-axis 3-position sun tracker," Solar Energy, vol. 85, no. 9, pp. 1935-1944, 2011.

[33] T. P. Chang, "Output energy of a photovoltaic module mounted on a single-axis tracking system," Applied energy, vol. 86, no. 10, pp. 2071-2078, 2009.

\section{Creative Commons Attribution License 4.0 (Attribution 4.0 International, CC BY 4.0)}

This article is published under the terms of the Creative Commons Attribution License 4.0

https://creativecommons.org/licenses/by/4.0/deed.en US 